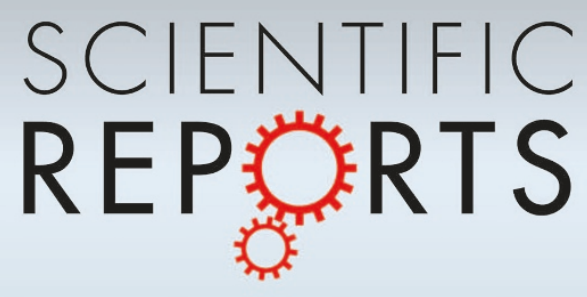

\title{
Multicellular Self-Assembled Spheroidal Model of the Blood Brain Barrier
}

SUBJECT AREAS:

BLOOD-BRAIN BARRIER

TRANSLATIONAL RESEARCH

PHARMACEUTICS

CELLULAR NEUROSCIENCE

Received

9 November 2012

Accepted

6 March 2013

Published

20 March 2013

Correspondence and requests for materials should be addressed to

P.-O.F. (per-ola.

freskgard@roche.com)

* These authors contributed equally to this work.

\author{
Eduard Urich ${ }^{1 *}$, Christoph Patsch ${ }^{2}$, Stefan Aigner' ${ }^{1}$ Martin Graf ${ }^{2}$, Roberto lacone ${ }^{3} \&$ Per-Ola Freskgård'
}

\begin{abstract}
'Pharma Research and Early Development, Neuroscience DTA, F. Hoffmann-La Roche Basel, Switzerland, ${ }^{2}$ Pharma Research and Early Development, Discovery Technologies, F. Hoffmann-La Roche Basel, Switzerland, ${ }^{3}$ Pharma Research and Early Development, CV \& Metabolism, F. Hoffmann-La Roche Basel, Switzerland.
\end{abstract}

The blood brain barrier (BBB) has evolved unique characteristics such as dense coverage of the endothelial cells by pericytes and interactions with astrocytes through perivascular endfeet. We study BBB formation in a 3-dimensional multicellular spheroid system of human primary brain endothelial cells (hpBECs), primary pericytes (hpPs) and primary astrocytes (hpAs). We show for the first time that hpBECs, hpPs and hpAs spontaneously self-organize into a defined multicellular structure which recapitulates the complex arrangement of the individual cell types in the BBB structure. Pericytes play a crucial role mediating the interaction between hpBECs and hpAs. This process is not dependent on a scaffold support demonstrating that formation and cellular architecture of the BBB is intrinsically programmed within each specific cell type. In a matrigel setup the hpBECs, hpPs and hpAs also undergo self-arrangement to form endothelial tube-like structures tightly covered by hpPs and loosely attached hpAs mainly at the junctions.

T he Blood Brain Barrier (BBB) is a highly evolved microvasculature system composed of different specialized cell types including brain endothelial cells (BECs) lining the lumen brain vasculature ${ }^{1}$, pericytes embedded within the abluminal basement membrane ${ }^{2,3}$, and astrocytes with their endfeet directly contacting the other cell types ${ }^{4}$. These three cell types together with the neurons, form what is termed the neurovascular unit $(\mathrm{NVU})^{5}$. It is established that one of the important determinants of BEC differentiation and functionality is this multicellular local environment within the BBB and particularly the direct interaction with surrounding cells and the extracellular matrix ${ }^{6,7}$. One important function of the $\mathrm{BBB}$ is to regulate exchange of substances between the blood and brain, and this is controlled by two very different mechanisms. First, the tight junctions between adjacent BECs form the basic structure to limiting paracellular permeability ${ }^{8}$ and secondly, transporters and receptors at the lumen and abluminal side of the BECs regulate transcellular transport ${ }^{9}$.

In a 2-dimensional (2D) in vitro BBB model BECs dedifferentiate spontaneously and rapidly lose BBB-like properties, acquiring a more generic endothelial cell phenotype, an event known as "phenotypic drift", addition, many of the current in vitro BBB models fail to address the three-dimensional (3D) cellular organization required for proper cellular differentiation, positioning effects and polarization and most models do not address the importance of direct cell-cell interactions. Consequently, this could explain some of the discrepancies in translation between in vitro and in vivo when studying the $\mathrm{BBB}^{1,10}$. For instance, this is the case when relatively high concentrations of compounds are used in vitro, where present cell models may be unable to quantify active transport due to poor expression of receptors ${ }^{11}$. Also, lack of cell surface receptor expression ${ }^{6}$ and alterations in intracellular trafficking and efficiency ${ }^{2}$, which are directly dependent on a native environment, make studies of receptor-mediated transport mechanisms in vitro less meaningful in current 2D BBB models.

We reasoned that studying the BBB in vitro would require the development of novel experimental multicellular culture systems that could replicate some of the key features of the in vivo microvasculature environment. This led us to develop a multicellular spheroidal BBB (MCS-BBB) model. Spheroid models of tumor cells and primary cells have been widely used ${ }^{12,13}$, to study the mechanisms of organogenesis ${ }^{14}$, liver physiology ${ }^{15}$, high-throughput toxicity measurements ${ }^{16}$ and cellular viral infectivity ${ }^{17}$. The MCS-BBB model consists of a mixed culture of human primary BECs (hpBECs), human primary pericytes (hpPs) and human primary astrocytes (hpAs). Here we show for the first time an in vitro 3D BBB model where all cell types are in direct contact with each other. The self-assembling process is spontaneous and intrinsically programmed within each cell type. Notably, the formation of the MCS-BBB structure is completely independent of any scaffold support where the three different cell types directly determines all interactions. Moreover, the pericytes play a central role in formation of these ' $\mathrm{BBB}$ balls' by mediating the interaction between the BECs and the astrocytes. We also find evidence that the 
cell-cell communication is much more pronounced in the MCS-BBB setup compare to a standard trans-well model.

\section{Results}

Directed self-alignment of neurovascular cells into ordered micro vascular tubes. Under favorable conditions, endothelial cells (ECs) seeded on a basement membrane undergo self-organization into structures that resemble capillary-like tubes ${ }^{18}$. The matrigel system that resemble the complex extracellular environment, also supports the ECs to replicate many of the important steps involved in angiogenesis ${ }^{19,20}$. We have applied the matrigel assay for studying the cellular interaction of hpBECs, hpPs and hpAs. First, the purity of all primary cell types was confirmed using cell-specific markers such as CD31 and CD144 for hpBECs (Fig. 1A), CD140b for hpPs (Fig. 1B) and GFAP for hpAs (Fig. 1C). All three human primary cell types showed purity higher than $99 \%$ and had cell type specific morphology when grown on a collagen I coated 2D surface (see Supplementary Fig. S1A-C online). All cell types were separately labeled with green (hpBECs), red (hpPs) or blue (hpAs) fluorescent long-term cell labeling reagents in order to track them, particularly when the cells were cultured together. After the labeling step, cells were seeded on a matrigel matrix to initiate proliferation. Expectedly, the single cultured hpBECs formed a branched tubular network connected by multicellular junctions within 18 hours post seeding (see Supplementary Fig. S1D online). Basal endothelial growth medium supplemented with recombinant human vascular endothelial growth factor (VEGF) and human serum was sufficient for inducing the formation of these EC capillary-like tubes. In contrast, neither hpPs nor hpAs alone or together were able to form tube-like structures, demonstrating that ECs are crucial for driving the tube formation in this setup (see Supplementary Fig. S1E-G online).

Dynamic interactions between hpBECs, hpPs and hpAs were studied by mixing the pre-labeled cells and subsequently seeding the mixture on the matrigel matrix. This assay permits free cell migration and unhindered cell-cell interactions, thereby supporting directed self-organization stimulated by all three cell types. The morphology and localization of each cell type were studied 18 hours post seeding. The hpBECs again formed tube-like structures in the presence of both hpPs and hpAs. Notably, an active recruitment of hpPs and hpAs occurred (Fig. 1D), which appeared to be specific, with an alignment of the cells both to the tubular network and the multicellular junctions. The hpPs primarily align along the hpBEC tubelike structures, and are often evenly spaced (Fig. 1D2, indicated by arrows). Similar arrangement of pericytes along the perivascular space has been demonstrated in vivo ${ }^{21}$. The brain vasculature is densely covered by pericytes, with a $1: 1-3: 1$ ratio between ECs and pericytes, and the abluminal surface is covered by pericytes to approximately $30 \%^{22}$. On the contrary, the hpAs only loosely align as rounded cells, preferably at the junctions where the different cell types significantly interact with each other (Fig. 1D4, indicated by arrows). In cases where the hpAs interact with the EC tubes, a pericyte is almost always in the vicinity. Moreover, hpPs co-cultured with a

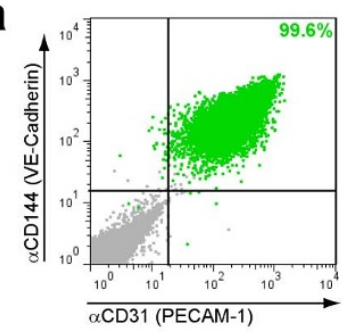

b

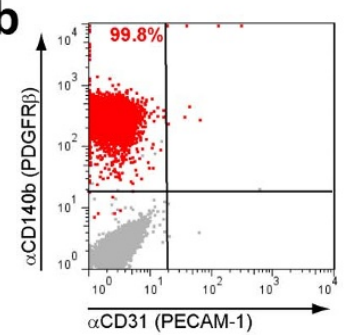

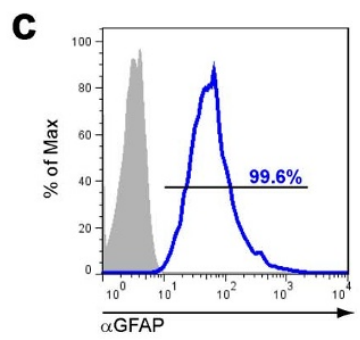
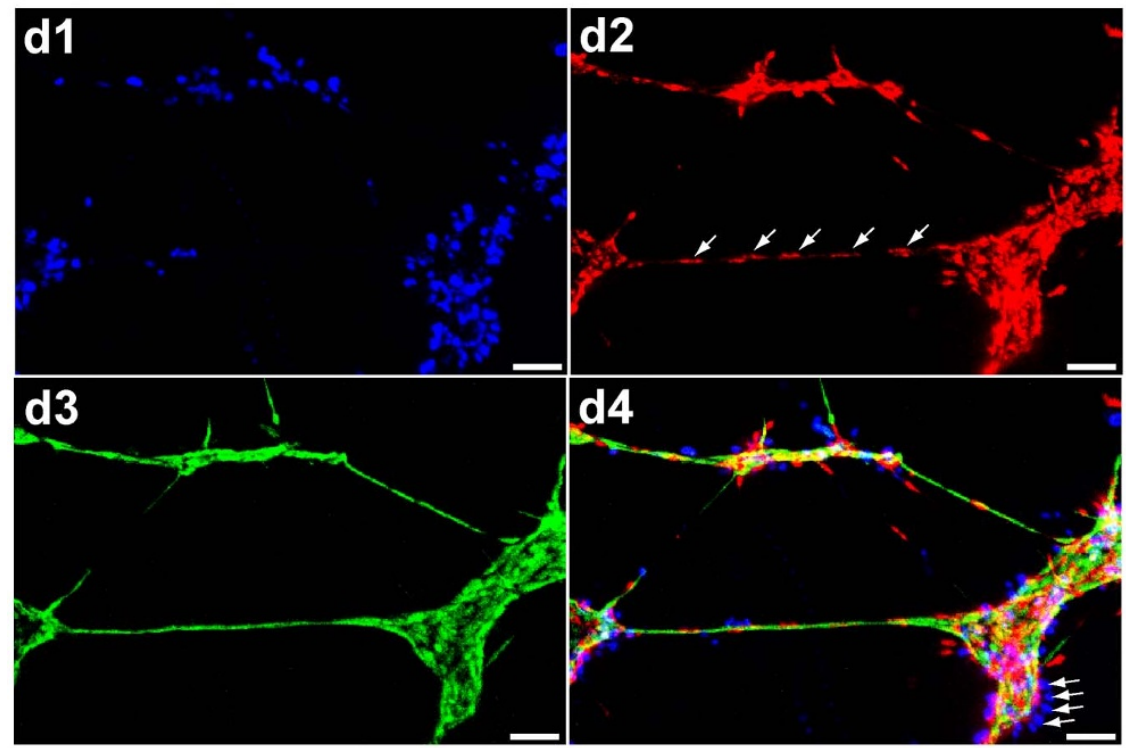

Figure 1 Brain endothelial tube formation model for studying spontaneous neurovascular unit cell assembly. (a-c) Flow cytometry analysis of confluent (a) hpBECs (green), (b) hpPs (red) and (c) hpAs (blue). Staining was done with indicated antibodies or relevant isotypes (grey population, grey histogram). One representative experiment of three is shown. (d) Examination of hpBEC tube formation (green), hpPs (red) and hpAs (blue) tube association on matrigel matrix using different fluorescent markers', 18 hours after seeding. The images were taken as an overlay of five 1 um thick confocal sections. Single channels (D1-3) and the merged image of all colors (D4) are displayed. White arrows in the upper right red channel image (D2) indicate hpPs aligned along an hpBECs tube and the white arrows in the lower right merged image (D4) point out the loosely associated hpAs. Bar equals 100 um. 
hpBECs undergo a self-arrangement in which the majority of the hpPs align along the hpBECs tubes (see Supplementary Fig. S2 online), demonstrating that the hpAs are not essential for the tight alignment of pericytes alongside the hpBEC tubes.

Spontaneous self-organization of neurovascular cells into organized multicellular spheroids. Growing cells in multicellular 3D spheroids has for example been successfully used to study early embryologic development and regulation of early organogenesis ${ }^{14}$ as well as tumor cell growth ${ }^{12,23}$. We used the $3 \mathrm{D}$ spheroid culture setup to mimic the multicellular composition of the $\mathrm{BBB}$ and provide a milieu where cells can freely interact in order to promote the formation of critical cellular organizations of the neurovascular structure. Different cell permutations (mono-, di- and tri-culturing) were investigated to study self-arrangement and cell-cell interactions. In a mono-culture setup, hpBECs (Fig. 2A) and hpPs (Fig. 2B) cluster and form a spherical structure while under identical conditions hpAs are not able to form stable spheroids by themselves (data not shown). This indicates that BEC and pericytes are programmed to form tight cell-cell interfaces whereas astrocytes apparently lack this ability. Furthermore, hpBECs spontaneously self-assemble in a wellorganized structure in co-cultures with hpPs. Here, the hpBECs form the outer layer of the spheroids (Fig. 2C). When hpPs are cocultured with hpAs they support formation of an astrocytic core and the pericytes align as a monolayer on the surface (Fig. 2D). In contrast, mixed co-cultures of hpBECs and hpAs form irregular aggregates (data not shown), demonstrating that BECs are unable to promote the formation of an ordered core structure of astrocytes as was shown for the pericytes. Adding hpBECs to preformed hpPs hpAs spheroids induces the attachment of the hpBECs onto the surface forming a three cellular layer structure where the pericytes separate the other two cell types (Fig. 2E). Intriguingly, when all three cell types were mixed in a $1: 1: 1$ ratio, something very interesting happens, the cells begins to interact with each other and form organized round structures freely in solution. The hpBECs spontaneously form a surface monolayer and establish a tight cellcell interaction with the pericytes (Fig. 2F). The astrocytes move to the inside and form the core of the spheroid separated from the hpBECs by the pericytes. Clearly, the pericytes seem to play an important role promoting a well-organized spheroid which essentially replicates the native cell-cell organization in the BBB. Notably, under these conditions the size of the spheroids remained unchanged over days of culture only in the presence of the outer hpBECs layer whereas the hpPs - hpAs spheroids continually grow until necrotic areas are formed in the center (data not shown). Thus, the hpBECs seem to be able to control the proliferation rate for both the hpPs and hpAs encapsulated within the spheroids.

Alteration in cell surface adhesion and receptor expression in the multicellular spheroids. Pericytes reside at the interface between the endothelium and the surrounding brain parenchyma and play critical roles in structural organization, maturation and specialization of BECs ${ }^{2,3,8}$. Also, pericytes synthesize and release most elements of the basement membrane and extracellular matrix proteins ${ }^{24}$, which are thought to be critical for BBB stabilization and polarization $^{25}$. As the contact between BECs and pericytes is mediated by a variety of cell adhesion molecules (CAMs), we investigated the surface expression levels of selected CAMs on both the hpBECs and hpPs grown in a standard trans-well or in the MCS-BBB system.

After 3 days of mono or co-culturing on trans-well filters (Fig. 3A \& 4A) or as spheroids (Fig. 3B \& 4B), the cells were detached from the filter or dissociated in the spheroids and the surface expression levels of CAMs were determined by flow cytometry. The dissociated cells show two well separated population of cells positive for the expected cell type specific markers CD31 and CD140b (data not shown) The surface expression levels of six selected CAMs, namely intercellular adhesion molecule 1 (ICAM-1, CD54), melanoma cell adhesion molecule (MCAM, CD146), intercellular adhesion molecule 2
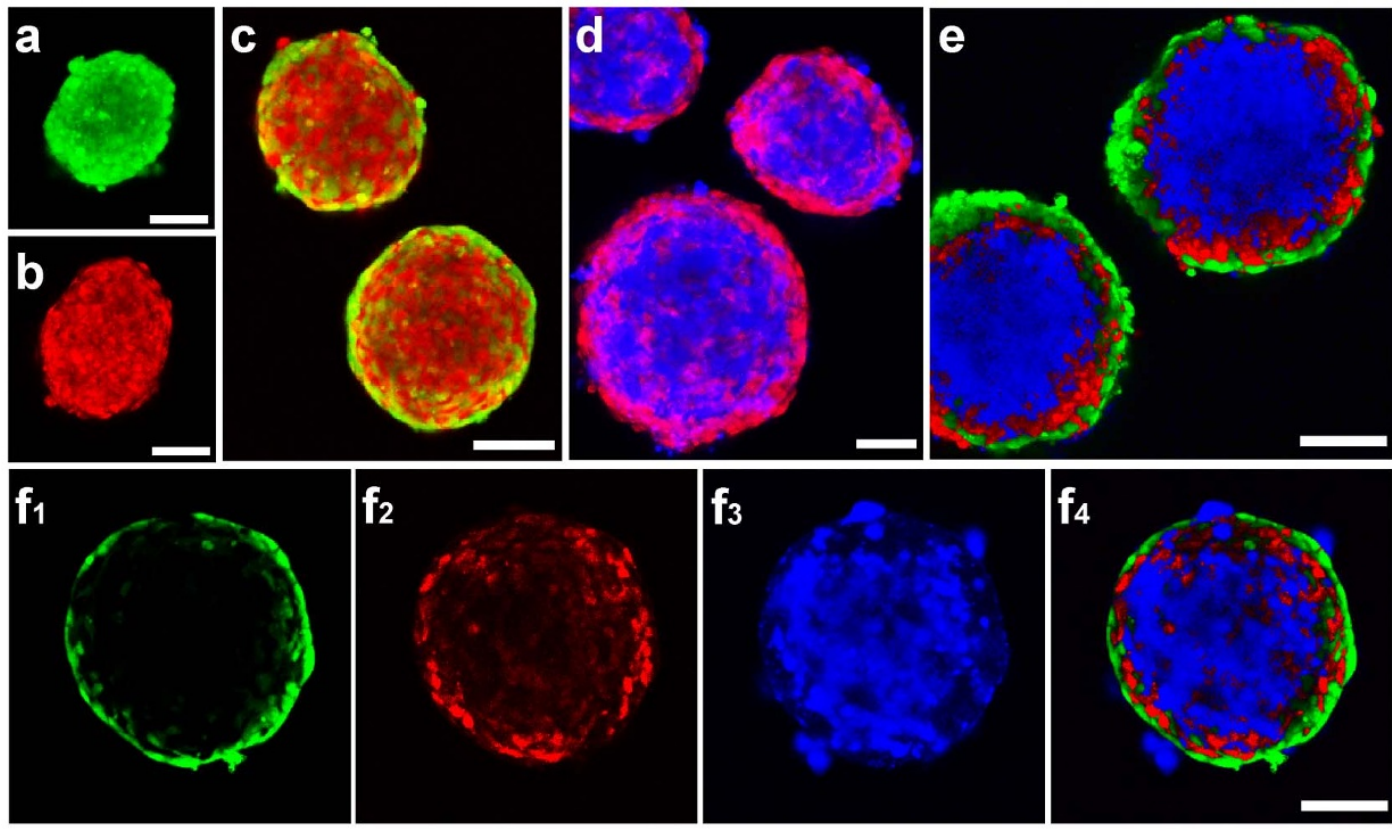

Figure 2 Spontaneous self-organization of co-culture spheroids of neurovascular unit cell types. Representative laser confocal images of fluorescently labeled hpBECs (green), hpPs (red) and hpAs (blue) single and co-culture spheroids. Cells were stained using cell tracker dyes for long term tracing of living cells. (a) hpBECs, (b) hpPs single-culture and (c) hpBEC/hpPs co-culture spheroids cultured for 3 days. (d) hpPs/hpAs co-culture spheroids cultured for 1 day prior to addition of (e) hpBECs for additional 2 days. The pictures were taken as an overlay of five 1 um thick confocal sections through the middle of the spheroid. A total of 20 spheroids in three independent experiments were analyzed for each culture condition. Bar equals 50 um. f1-f4 shows a spontaneously formed spheroid where the hpBECs is selectively shown in green (f1), hpPs selectively shown red (f2), hpAs selectively shown in blue (f3) and the complete spheroid (f4) composed of all the three different cell types. 
(ICAM-2, CD102), activated leukocyte cell adhesion molecule (ALCAM, CD166), platelet endothelial cell adhesion molecule 1 (PECAM-1, CD31) and VE-Cadherin (CD144) were investigated (Fig. 3 and 4). Confluent hpBECs grown alone in the trans-well system as a monolayer express all these CAMs (Fig. 3A), while the hpPs in mono culture only express ICAM-1 and ALCAM (Fig. 4A). The expression levels of all measured CAMs, except MCAM, were reduced when hpBECs were co-cultured with hpPs (Fig. 3A). On pericytes in co-culture with hpBECs, the ICAM-1 and ALCAM expression was reduced, while expression levels of MCAM were increased (Fig. 4A).

In our $3 \mathrm{D}$ spheroidal setup when the hpBECs were grown alone, surface expression was detected for all CAMs except ALCAM (Fig. 3B) while the hpPs in mono 3D culture only express ICAM-1 and ALCAM as in the trans-well system (Fig. 4B). The expression levels of all measured CAMs, except ICAM-2, were increased when hpBECs were co-cultured with hpPs (Fig. 3B). Interestingly, both PECAM-1 and VE-Cadherin expression on the hpBECs was induced by the pericytes. This potentially indicates that extensive coverage of pericytes could enhance the interactions between the BECs by boosted adherent junction expression and thereby support a stable tight junction organization. For the pericytes in co-culture with hpBECs a strong reduction of ICAM-1 and ALCAM was noted whereas for MCAM there was a marked induction in surface expression levels (Fig. 4B). In addition, two other cell surface markers, PDGFRa and PDGFRb were investigated. Expression of these receptors was as expected undetectable on the hpBECs both in presence and absence of pericytes. On the other hand, both receptors were clearly detected on isolated hpPs, especially the PDGFb isoform (Fig. 3). In co-culture with hpBECs, the low but detectable levels of PDGFRa were completely down-regulated whereas the high surface expression levels of PDFRb on hpPs were only slightly reduced by hpBECs co-culturing (Fig. 4). These data show that PDGFb is a major isoform on pericytes in our system and the cell surface levels are not substantially affected by neighboring BECs in direct contact.

Next we investigated changes in receptor expression on the hpBECs in absence and presence of hpPs and hpAs, both in the trans-well and the 3D spheroidal setup. For the 3D spheroidal experiment we dissociated the cells and separated them based on EC (CD31) and a pericyte (CD140b) specific markers (Fig. 5A). All investigated cell types were identified as distinct populations in the tri-culture setup, about $47 \%$ were pericytes, $27 \%$ were astrocytes and
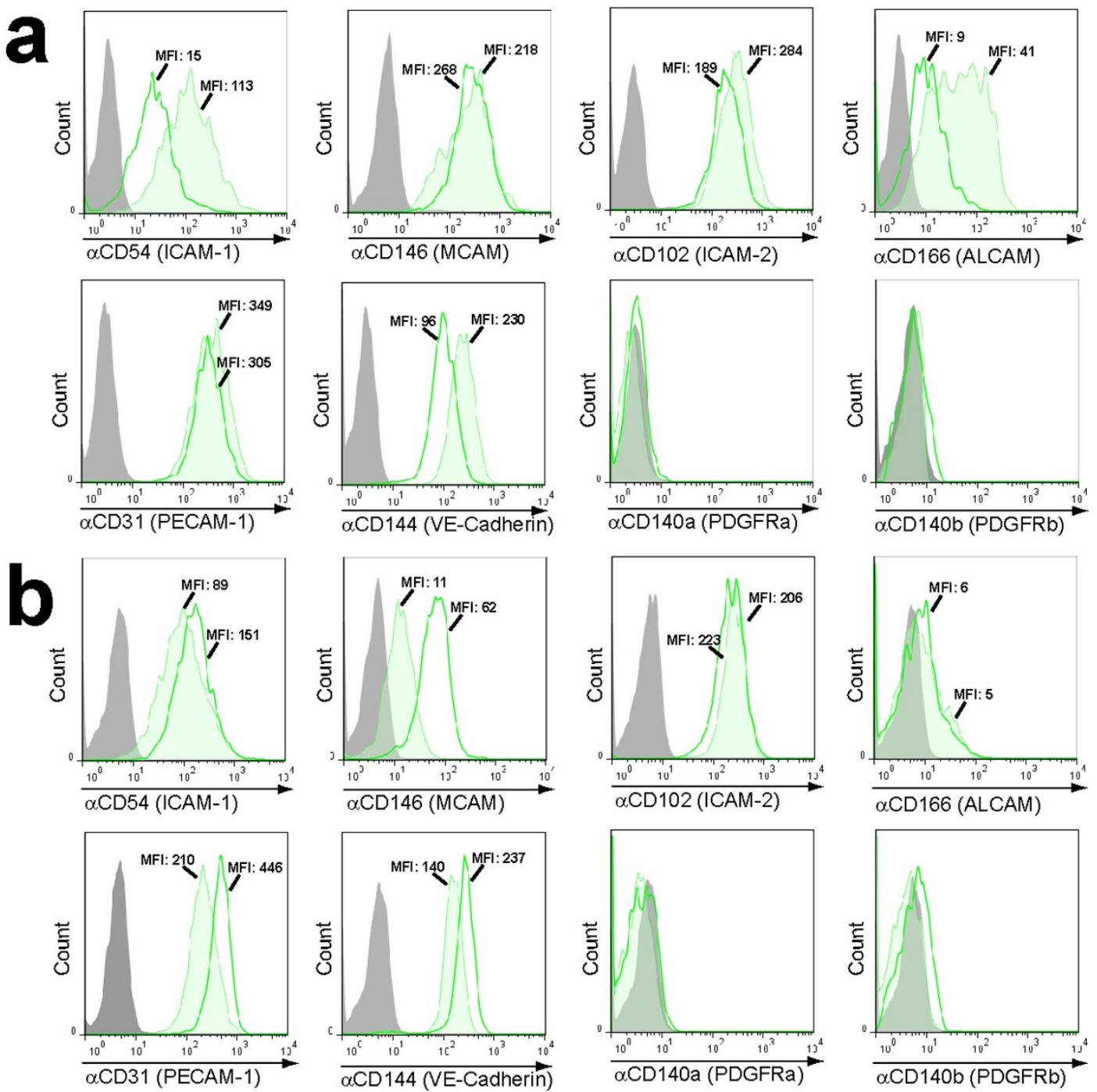

Figure 3 Pericytes differently affect the surface expression of cellular adhesion molecules on brain microvascular endothelial cells. Flow cytometry analysis of CD31 pre-gated hpBECs grown on (a) trans-well filters and as (b) spheroids. Single cultured hpBECs (tinted histogram) or hpBECs co-cultured with hpPs (open histogram) for 3 days were stained with indicated antibodies or relevant isotypes (grey histogram). Data are representative of two or more experiments with 1000 spheroids or 3 trans-well filters, respectively, per individual staining. (MFI $=$ mean fluorescence intensity). 

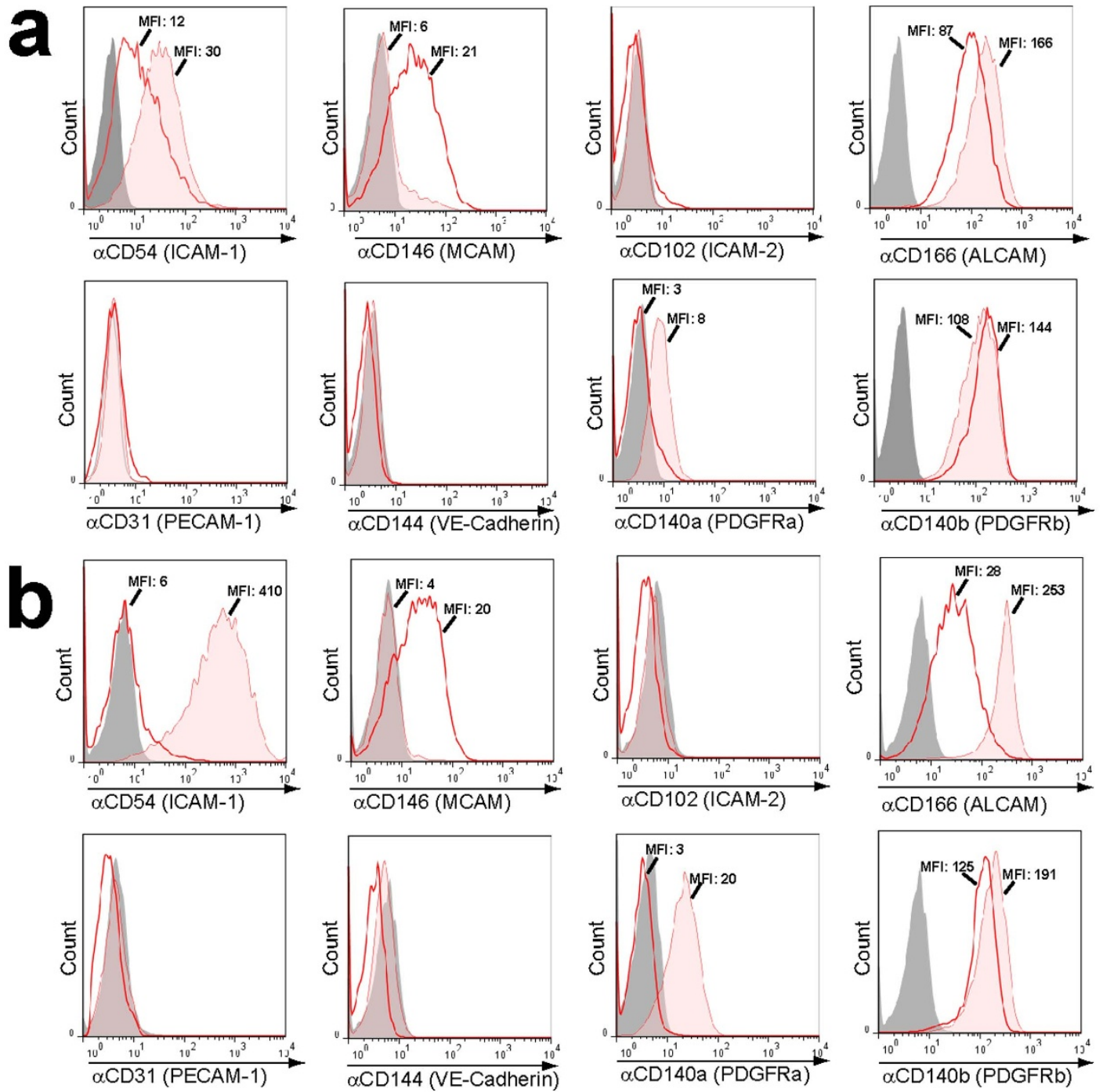

Figure $4 \mid$ Brain microvascular endothelial cells strongly modulate cellular adhesion molecules surface expression on pericytes. Flow cytometry analysis of CD140b pre-gated hpPs grown on (a) trans-well filters and as (b) spheroids. Single cultured hpPs (tinted histogram) or hpPs co-cultured with hpBECs (open histogram) for 3 days were stained with indicated antibodies or relevant isotypes (grey histogram). Data are representative of two or more experiments with 1000 spheroids or 3 trans-well filters, respectively, per individual staining. (MFI = mean fluorescence intensity).

$17 \%$ were endothelial cells (Fig. 5A). One described BBB receptor is the insulin receptor (IR). It has earlier been shown that insulin transport by BECs is increases in the presence of pericytes ${ }^{26}$, possibly indicating that pericytes regulate the level of the IR expression on endothelial cells. In our 3D spheroidal culture setup, where the hpBECs are in direct cell-cell contact with the hpPs, there is a profound increase in the expression level of the IR, which was even more enhanced in the triple 3D spheroidal system also including the hpAs (Fig. 5B). Another described BBB receptor, the leptin receptor (LR) was decreased in the presence of both hpPs and hpAs in the $3 \mathrm{D}$ spheroidal system (Fig. 5B). A previous study has shown that leptin transport over a BEC monolayer is significantly decreased in presence of C6 astrocytoma cells ${ }^{27}$. This finding is in agreement with our data showing that astrocytes reduce LR expression, thereby likely lowering leptin transport. For the IGF receptor we see an increase in expression on the hpBECs in spheroidal co-culturing with hpPs which is not further enhanced by the presence of hpAs (Fig. 5B). Another typical BBB receptor is the transferrin receptor (TfR). The experiment using the 3D spheroid system clearly shows that pericytes down-regulate the cell surface expression of TfR on BECs (Fig. 5B). In contrast, in the trans-well setup there was no change in expression levels of any of these receptors by the presence of either hpPs or hpAs grown under identical conditions used in the spheroidal model (Fig. 5C). This is likely due to the absence of, or very small (through the pores in the filter), contact between the cells in this system.

\section{Discussion}

The complex interplay of endothelial cells, pericytes, and astrocytes in the cerebral microvasculature generates a sophisticated multicellular architecture that controls both paracellular and transcellular exchange between the blood and the brain. Although BECs are the principal cell type of the $\mathrm{BBB}$, with unique barrier properties and transport functions, pericytes and astrocytes evidently play an important indirect regulatory role. Pericytes have been suggested to be involved in controlling intracellular trafficking within the $\mathrm{BECs}^{2}$, integrity during $\mathrm{BBB}$ embryogenesis ${ }^{3}$, regulation of blood flow $^{28,29}$ and to have an instructive role in angiogenesis ${ }^{30}$. Similarly, astrocytes have been implicated in multiple functions such as the exchange of molecules between blood and brain parenchyma ${ }^{22}$, regulation of tight junctions ${ }^{31}$ and affecting the expression levels of adhesion molecules on the BECs ${ }^{6,32}$. Recently, it was demonstrated that 

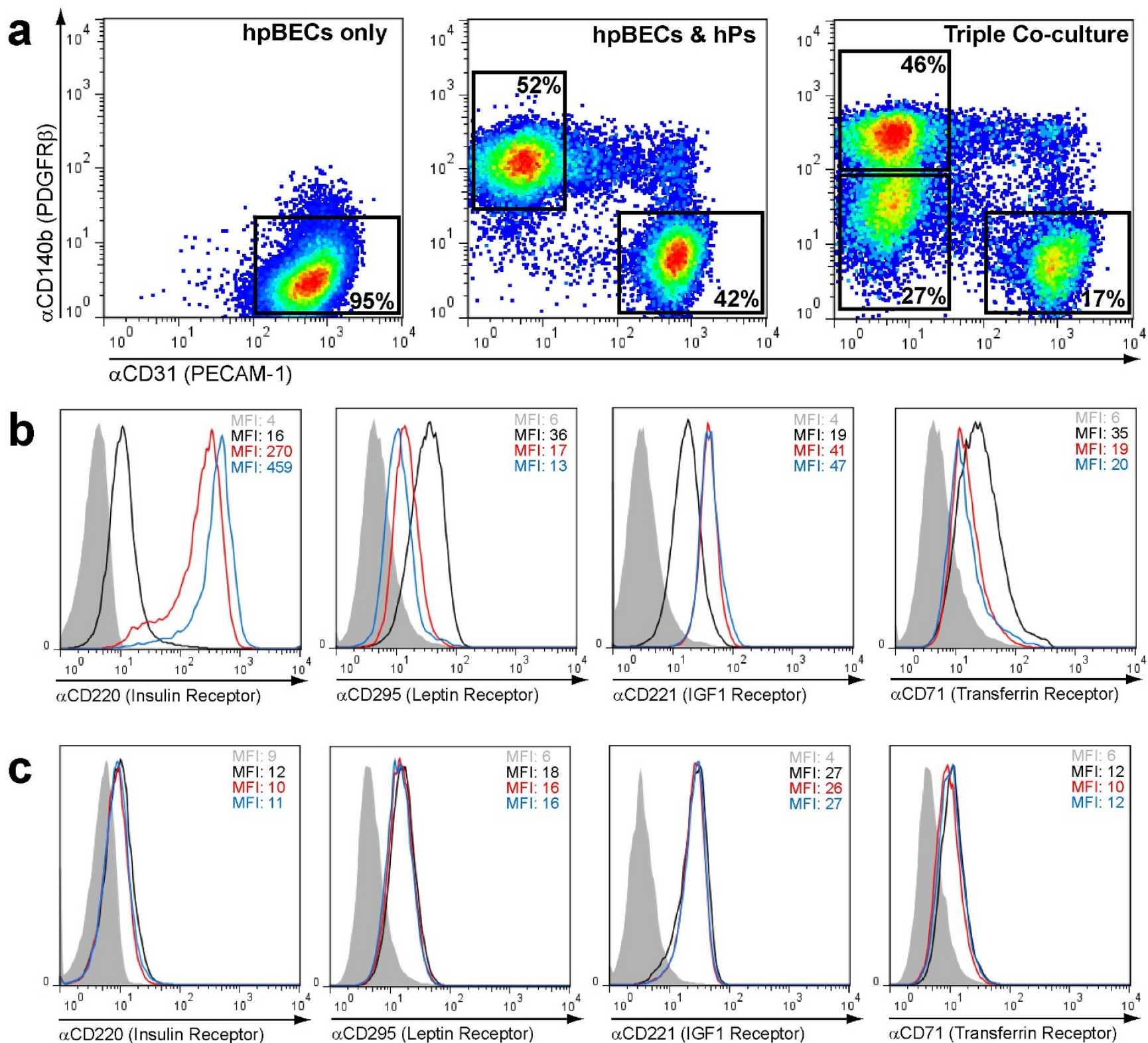

Figure 5 Direct contact co-culture of neurovascular unit cells alter receptor expression on brain microvascular endothelial cell. HpBECs single cultures, hpBECs/hpPs and hpBECs/hpPs/hpAs co-cultures grown as (a \& b) spheroids or (c) in the trans-well set-up for 3 days were stained with indicated cell type specific antibodies and analyzed by flow cytometry. (b)Spheroid derived CD31+ \& CD140b- gated hpBECs or (c) hpBECs harvested from the trans-well inserts were further examined for the indicated receptor expression. (b \& c) Surface receptor expression levels on hpBECs are displayed for single hpBECs culture (black histogram), co-cultured with hpPs (red histogram) or triple co-cultured with hpPs and hpAs (blue histogram). Data are representative of two experiments with 1000 spheroids respectively 3 trans-well filters per individual staining. (MFI = mean fluorescence intensity).

astrocytes could regulate CSF entry into the brain the parenchyma via water channel aquaporin-4 (AQP4) ${ }^{33}$.

However, the role of BECs, pericytes and astrocytes in BBB assembly is less well understood. Therefore, we have adopted the matrigel and 3D hanging-drop spheroid models to study inherent association properties between these key cells. These models have been compared to a more standard trans-well system which was developed primarily due to the convenience to study permeability over a BECs monolayer, but is much less relevant from a biological standpoint (Fig. 6). Our novel MCS-BBB model is, to our knowledge, the first in vitro model that allows the individual cell types to spontaneously self-organize without any type of scaffold support provided. In this model, the cells form a well-defined multicellular structure which recapitulates some of the complex arrangement of the individual cell types of the BBB. Evidently, the driving force for BBB formation is inherently encoded within each cell type. Pericytes have the unique functionality to interact both with endothelial cells and astrocytes and are essential for mediating the interaction between these two cell types to form a multi-cellular layer. This is also observed in the matrigel experiment (Fig. 1D4) where the astrocytes attach to the endothelial tubes only by the facilitating role of the pericytes, either directly via cell-cell contacts or possibly in close vicinity. Endothelial cells and pericytes are 'adhesion prone cells' forming regular $3 \mathrm{D}$ structures on their own or in presence of each other (Fig. 2A and 2B). Interestingly, our observation that pericytes but not endothelial cells can induce astrocyte cell structure regularity suggests that endothelial cells have low levels of interaction capacities with the astrocytes at the abluminal side. The importance of the $3 \mathrm{D}$ architec- 


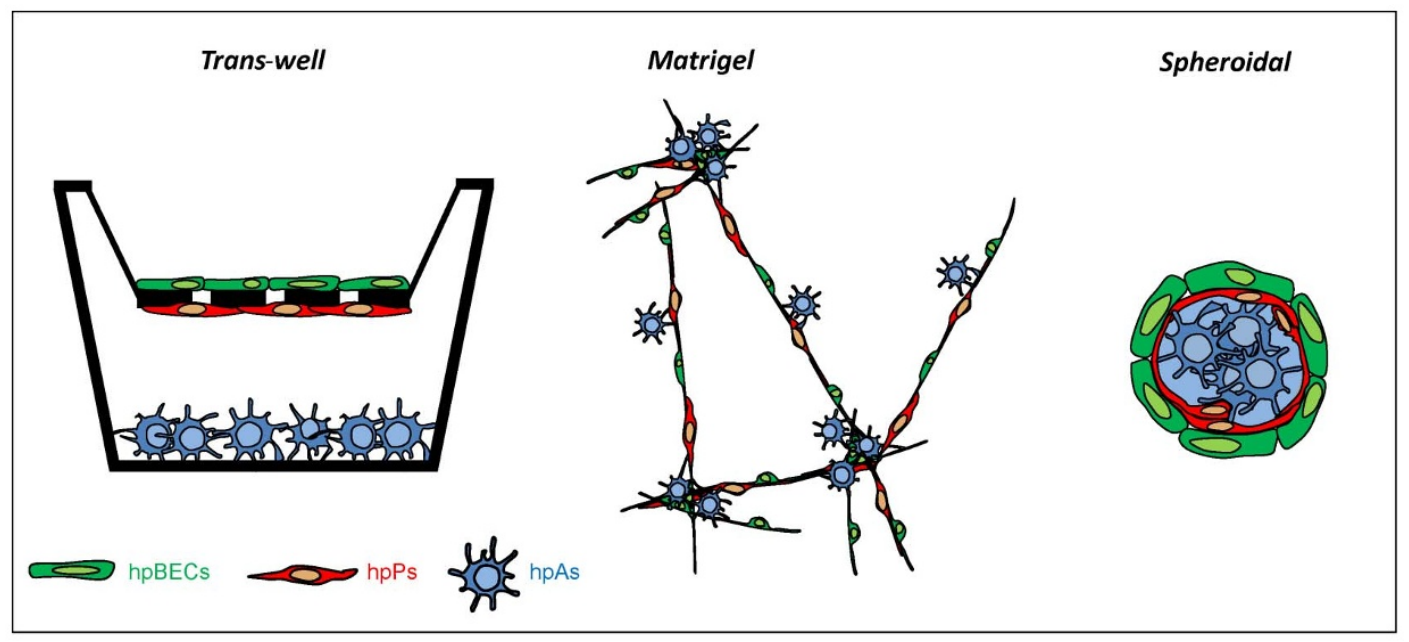

Figure 6 Schematic overview of the three different BBB model investigated in this study. (Trans-well): Is currently the standard setup where the BECs are grown on a filter insert with small pores to allow permeability studies between the two chambers. In co-culturing conditions, pericytes in normally grown on the opposite side of the filter potentially allow some cell-cell interaction through the pores and the astrocytes are grown in the bottom of the lower chamber to possibly influence the other cells by secreted factors into the media. (Matrigel): Cells are seeded in a gel structure to support migration an assembling. In this setup the BECs form tube-like structures on which pericytes preferentially adhere to, while the astrocytes are more loosely attached to the cell junctions. (Spheroidal): This model is completely independent of any supportive scaffold material for cell organization. The cells are allowed to freely self-assemble based on the intrinsic properties of each cell type. In our novel MCS-BBB setup, three key cell types of the neurovascular unit spontaneously form the spheroidal structure. The BECs form an outer cell monolayer representing the lumen side. The astrocytes accumulate in the core of the spheroids that could be perceived as the parenchyma space. Finally, pericytes line up in between to mediate a three cellular layer arrangement reproducing the morphological arrangement of the different cell types in the BBB.

ture of the MCS-BBB manifests itself in the responsiveness and cellular adaptation of the BECs in this configuration. There are significant alterations in expression levels of cell surface receptors on the endothelial cells induced primarily by the presence of pericytes, which is not detected in a standard trans-well setup (Fig. 5). Thus, this once more illustrates the importance of extensive and direct contacts between the cells and potentially also underscores the requirement of a scaffold free system. One of the most fascinating findings in this study was the observation that the cells spontaneously self-assemble in the spheroids, reproducing the morphological arrangement of the different cell types in the BBB. The endothelial cells form an outer monolayer and the astrocytes accumulate in the center of the spheroids while the pericytes line up in between mediating a three cellular layer arrangement. Our results suggest a crucial role for the pericytes in the assembling process by possessing the unique capacity to productively interact both with the endothelial cells and the astrocytes. The pericytes interact with the endothelial cells and astrocytes separately or together with both cell types.

Taken together, our results demonstrate that major progress could be made in studying the BBB in vitro by allowing the key cells to freely and spontaneously interact. Here we have elucidated several essential principles in cell-cell communication and interactions within the BBB. This knowledge will support further maturation of this methodology, especially for studying the functional aspects of the cells in this $3 \mathrm{D}$ configuration such as tight junction formation and transcellular activities. A next logical step is to transfer this format into a device to allow us to study complex BBB transport mechanisms and to test drugs and brain drug-delivery strategies that target the cerebrovascular network.

\section{Methods}

Cell culture conditions. Human cerebral astrocytes (hpAs) and human brain vascular pericytes (hpPs) were acquired from ScienCell, USA. Primary Human Brain Microvascular Endothelial Cells (hpBECs) were purchased from Applied Cell Biology Research Institute, USA. HpBECs were grown in endothelial basal medium-2 (EBM2) (Lonza, UK) supplemented with EGM-2 (Lonza, UK). HpPs were grown in pericyte growth supplemented medium (ScienCell, USA) and hpAs were grown in astrocyte growth supplemented medium (ScienCell, USA). The hpBECs, hpPs and hpAs were used in the experiments between passage 2 and 4 . For the spherical and matrigel matrix co-cultivation, cells were grown in EBM-2 supplemented with $2 \%$ human serum (Blood Bank, Basel, Switzerland) and $50 \mathrm{ng} / \mathrm{ml}$ rhVEGF (Preprotech). Immediately before using the cells for the matrigel or spheroid culture the hpBECs were stained green, the hpPs were stained red and the hpAs were stained blue by using Cell Tracker dyes from Invitrogen (Carlsbad, CA) according to the manufacturer's instructions. All culture ware (BD Falcon) and transwell filters (Millipore) (pore size $0.4 \mu \mathrm{m}$, high density pores) were coated with rat tail collagen type I solution (BD Bioscience) at a concentration of $10 \mu \mathrm{g} / \mathrm{ml}$ according to the manufactures instructions. Cells were cultured in the incubator at $37^{\circ} \mathrm{C}$ with $5 \% \mathrm{CO} 2,95 \%$ fresh air and saturated humidity.

Matrigel culture. For the matrigel tube formation and association assays the hpBECs, $\mathrm{hpPs}$ and hpAs were grown using the in vitro angiogenesis a kit from AMS biotechnology (Lugano, Switzerland). The hpBECs were seeded at a density of $2 \times 10^{4}$ cells $/ \mathrm{cm}^{2}$ ) in the monoculture experiments. For co-culture experiments hpBECs, hpPs and hpAs were cultured in ratios $2: 1: 1$.

Spheroid culture. Hanging Droplet Culture Plates (HDCPs) (Roche, Switzerland) were used to generate mono- and co-cultured spheroids of $1000 \mathrm{hpBECs}, 1000 \mathrm{hpPs}$ and 1000 hpAs. The HDCPs design is stabilizing the cell medium suspension and enables to additionally add volume of medium or cells to the preexisting droplet. $70 \mathrm{ul}$ of the cell suspension supplemented with $4 \%$ Methylcellulose (Sigma), was dispensed into each well of the 24well HDCPs. Plates were then inverted and incubated under standard conditions for 72 hours. Alternatively, $25 \mathrm{ul}$ of the hpPs/hpAs cell suspension was dispensed into each well of the 24well HDCPs and 24 hours later the HDCPs were up-righted and $1000 \mathrm{hpBECs}$ were introduced in $45 \mathrm{ul}$ of the same media. These cultures were then re-inverted and further incubated for 48 hours. Spheroids were harvested, collected by gravity and either fixed (2\% PFA) (SigmaAldrich) or dissociated ( $0.05 \%$ Trypsin) (Gibco). Imaging was performed using confocal laser scanning microscopy (Leica) or flow cytometry (Millipore). The Methylcellulose used for these experiments was diluted from a stock solution that was generated by dissolving $6 \mathrm{~g}$ of Methylcellulose in $500 \mathrm{ml}$ of EBM-2. After centrifugation the clear, gel-like supernatant was used for the experiments. Methylcellulose prevents adhesion of cells and acts as an inert viscosity modulation substance.

Trans-well culture. The hpPs were seeded $\left(1 \times 105\right.$ cells $\left./ \mathrm{cm}^{2}\right)$ on the bottom side of rat tail collagen coated transwell inserts (Millipore) and the hpAs $\left(1 \times 105 \mathrm{cells} / \mathrm{cm}^{2}\right)$ were seeded on collagen I coated 12 well plates. The cells were let to adhere for 2 hours, then the hpBECs $\left(1 \times 105\right.$ cells $\left./ \mathrm{cm}^{2}\right)$ were seeded on the upper side of the likewise collagen I coated inserts containing no cells or hpPs. The inserts were subsequently placed in the wells of the 12-well culture plates containing no cells or hpAs. 
Flow cytometry analysis. All antibodies were directly labeled and purchased from $\mathrm{BD}$ Pharmingen and R\&D Biosystems. After the staining the cells were washed twice with stain buffer (BD Pharmingen) and analyzed using a Guava easyCyte flow cytometer (Millipore). For surface staining, $1 \times 106$ cells were pelleted and incubated for 45 minutes at $4^{\circ} \mathrm{C}$ with the following antibodies: anti-CD31 (WM59), anti-CD140b (PR7212), anti-CD140a (PRa292), anti-CD54 (HA58), anti-CD144 (55-7H1), anti-CD102 (CBR-1C2/2), anti-CD166 (3A6), anti-CD146 (P1H12) anti-CD220 (3B6/IR), anti-CD295 (52263), anti-CD221 (1H7), anti-CD71 (M-A712), IgG1 isotype ctr. (MOPC-21), IgG2a isotype ctr. (G155-178) and IgG2b isotype ctr. (2735). For intracellelular staining $1 \times 106$ pelleted hpACs were fixed (BD Cytofix, BD Pharmingen) for at $37^{\circ} \mathrm{C}$ for 10 minutes, permeabilized (BD Phosflow Perm III) on ice for 30 minutes and incubated with the following: anti-GFAP (1B4).

1. Cecchelli, R. et al. Modelling of the blood-brain barrier in drug discovery and development. Nat Rev Drug Discov 6, 650-661 (2007).

2. Armulik, A. et al. Pericytes regulate the blood-brain barrier. Nature 468, 557-561 (2010).

3. Daneman, R., Zhou, L., Kebede, A. A. \& Barres, B. A. Pericytes are required for blood-brain barrier integrity during embryogenesis. Nature 468, 562-566 (2010).

4. Abbott, N. J., Ronnback, L. \& Hansson, E. Astrocyte-endothelial interactions at the blood-brain barrier. Nat Rev Neurosci 7, 41-53 (2006).

5. Hawkins, B. T. \& Davis, T. P. The blood-brain barrier/neurovascular unit in health and disease. Pharmacol Rev 57, 173-185 (2005).

6. Urich, E., Lazic, S. E., Molnos, J., Wells, I. \& Freskgard, P. O. Transcriptional profiling of human brain endothelial cells reveals key properties crucial for predictive in vitro blood-brain barrier models. PLoS One 7, e38149 (2012).

7. Lyck, R. et al. Culture-induced changes in blood-brain barrier transcriptome: implications for amino-acid transporters in vivo. J Cereb Blood Flow Metab 29, 1491-1502 (2009).

8. Winkler, E. A., Bell, R. D. \& Zlokovic, B. V. Central nervous system pericytes in health and disease. Nat Neurosci 14, 1398-1405 (2011).

9. Zlokovic, B. V. The blood-brain barrier in health and chronic neurodegenerative disorders. Neuron 57, 178-201 (2008).

10. Neuwelt, E. et al. Strategies to advance translational research into brain barriers. Lancet Neurol 7, 84-96 (2008).

11. Hakkarainen, J. J. et al. Comparison of in vitro cell models in predicting in vivo brain entry of drugs. Int J Pharm 402, 27-36 (2010).

12. Hirschhaeuser, F. et al. Multicellular tumor spheroids: an underestimated tool is catching up again. J Biotechnol 148, 3-15 (2010).

13. Brophy, C. M. et al. Rat hepatocyte spheroids formed by rocked technique maintain differentiated hepatocyte gene expression and function. Hepatology 49 , 578-586 (2009).

14. Ungrin, M. D., Joshi, C., Nica, A., Bauwens, C. \& Zandstra, P. W. Reproducible, ultra high-throughput formation of multicellular organization from single cell suspension-derived human embryonic stem cell aggregates. PLoS One 3, e1565 (2008).

15. Sakai, Y., Yamagami, S. \& Nakazawa, K. Comparative analysis of gene expression in rat liver tissue and monolayer- and spheroid-cultured hepatocytes. Cells Tissues Organs 191, 281-288 (2010).

16. Tung, Y. C. et al. High-throughput 3D spheroid culture and drug testing using a 384 hanging drop array. Analyst 136, 473-478 (2011).

17. Andrei, G. Three-dimensional culture models for human viral diseases and antiviral drug development. Antiviral Res 71, 96-107 (2006).

18. Arnaoutova, I., George, J., Kleinman, H. K. \& Benton, G. The endothelial cell tube formation assay on basement membrane turns 20 : state of the science and the art. Angiogenesis 12, 267-274 (2009).

19. Davis, G. E., Koh, W. \& Stratman, A. N. Mechanisms controlling human endothelial lumen formation and tube assembly in three-dimensional extracellular matrices. Birth Defects Res C Embryo Today 81, 270-285 (2007).

20. Kleinman, H. K. \& Martin, G. R. Matrigel: basement membrane matrix with biological activity. Semin Cancer Biol 15, 378-386 (2005).
21. Armulik, A., Genove, G. \& Betsholtz, C. Pericytes: developmental, physiological, and pathological perspectives, problems, and promises. Dev Cell 21, 193-215 (2011).

22. Nuriya, M., Shinotsuka, T. \& Yasui, M. Diffusion Properties of Molecules at the Blood-Brain Interface: Potential Contributions of Astrocyte Endfeet to Diffusion Barrier Functions. Cereb Cortex online July 9 (2012).

23. Upreti, M. et al. Tumor-Endothelial Cell Three-dimensional Spheroids: New Aspects to Enhance Radiation and Drug Therapeutics. Transl Oncol 4, 365-376 (2011).

24. Ozerdem, U., Grako, K. A., Dahlin-Huppe, K., Monosov, E. \& Stallcup, W. B. NG2 proteoglycan is expressed exclusively by mural cells during vascular morphogenesis. Dev Dyn 222, 218-227 (2001).

25. Dejana, E. Endothelial cell-cell junctions: happy together. Nat Rev Mol Cell Biol 5, 261-270 (2004)

26. Nakaoke, R., Verma, S., Niwa, M., Dohgu, S. \& Banks, W. A. Glucose-regulated blood-brain barrier transport of insulin: Pericyte-astrocyte-endothelial cell cross talk. Int J Neuroprot Neuroregeneration 3, 195-200 (2007).

27. Hsuchou, H. et al. Role of astrocytic leptin receptor subtypes on leptin permeation across hCMEC/D3 human brain endothelial cells. J Neurochem 115, 1288-1298 (2010).

28. Peppiatt, C. M., Howarth, C., Mobbs, P. \& Attwell, D. Bidirectional control of CNS capillary diameter by pericytes. Nature $\mathbf{4 4 3}, 700-704$ (2006).

29. Hamilton, N. B., Attwell, D. \& Hall, C. N. Pericyte-mediated regulation of capillary diameter: a component of neurovascular coupling in health and disease. Front Neuroenergetics 2: 21 May (2010).

30. Dore-Duffy, P. \& LaManna, J. C. Physiologic angiodynamics in the brain Antioxid Redox Signal 9, 1363-1371 (2007).

31. Lippmann, E. S. et al. Derivation of blood-brain barrier endothelial cells from human pluripotent stem cells. Nat Biotechnol 30, 783-791 (2012).

32. Alvarez, J. I. et al. The Hedgehog pathway promotes blood-brain barrier integrity and CNS immune quiescence. Science 334, 1727-1731 (2011).

33. Iliff, J. J. et al. A Paravascular Pathway Facilitates CSF Flow Through the Brain Parenchyma and the Clearance of Interstitial Solutes, Including Amyloid beta. Sci Transl Med 4, 147ra111 (2012).

\section{Acknowledgments}

We thank A. Hayes and D. Wernet for technical assistance, T. Kissling for providing the HDCP plates and J. Rosenhauer for intellectual input. S.A. was supported by a Roche Postdoctoral Fellowship (RPF)

\section{Author contributions}

E.U. and C.P. designed and performed the experiments, analyzed and interpreted the data and wrote the paper; E.U., C.P., S.A., M.G., R.I. and P.-O.F. supervised the project and wrote the paper.

\section{Additional information}

Supplementary information accompanies this paper at http://www.nature.com/ scientificreports

Competing financial interests: All authors are under paid employment by the company $\mathrm{F}$. Hoffmann-La Roche.

License: This work is licensed under a Creative Commons

Attribution-NonCommercial-NoDerivativs 3.0 Unported License. To view a copy of this license, visit http://creativecommons.org/licenses/by-nc-nd/3.0/

How to cite this article: Urich, E. et al. Multicellular Self-Assembled Spheroidal Model of the Blood Brain Barrier. Sci. Rep. 3, 1500; DOI:10.1038/srep01500 (2013). 